\title{
Evaluating the Statistical Significance of a Fatigue-Life Reduction Due to Macro-Porosity
}

\author{
Ana Bižal*,1 - Jernej Klemenc ${ }^{2}$ - Matija Fajdiga² \\ ${ }^{1}$ Hella Saturnus Slovenija d.o.o., Slovenia \\ 2University of Ljubljana, Faculty of Mechanical Engineering, Slovenia
}

\begin{abstract}
This study focuses on an evaluation of the significance of the fatigue-life reduction due to macro-porosity present in pressure-die-casted aluminium specimens. Three statistical models, i.e., univariate analysis of variance, multivariate analysis of variance and linear regression with dummy variables, were applied to test the statistical significance of the fatigue-life reduction. The three statistical models were applied for the case of experimentally determined fatigue-life data for an AISi9Cu3 alloy with different levels of macro-porosity. Cylindrical specimens according to ASTM E606 were manufactured by pressure die casting using different manufacturing parameters (die pressure, die temperature) to artificially introduce detectable macro-pores into the specimens. The manufactured specimens were classified into three groups, representing their levels of porosity, which were identified based on x-ray images of the specimens. For each group, strain-controlled fatigue tests were performed at different strain levels. Of these approaches, linear regression with dummy variables proved to be the most appropriate, due to its ability to robustly identify the differences between the fatigue lives for different porosity levels.
\end{abstract}

Keywords: AISi9Cu3 alloy, porosity, fatigue life, ANOVA, MANOVA, linear regression with dummy variables

\section{INTRODUCTION}

Engineering applications usually require a homogeneous material; however, this is sometimes impossible to achieve. Alloys and castings, for example, are very susceptible to inhomogeneities. And while some inhomogeneities can be avoided completely, others, like porosity, can only be reduced. Despite many studies that have shown the detrimental effect of such inhomogeneities on fatigue life [1, $2]$, castings are still widely used in the automotive industry due to their favourable strength-to-weight ratio.

By knowing that inhomogeneities cannot be completely avoided, the question arises: when does the level of the inhomogeneities start to significantly influence the fatigue behaviour of the structures? In our study we have focused on the AlSi9Cu3 aluminium alloy, since it is often used in the automotive industry for complex cast parts such as engine supports. We narrowed our study to macroporosity inhomogeneities for a few reasons: a) it can be relatively easily introduced into specimens by varying the casting parameters, b) it can be detected using non-destructive inspections and c) it can be estimated with numerical simulations [3].

In the production process, when macro-porosity is discovered within a cast part, the part is normally discarded. This leads to a decreased throughput of good products as well as increased production costs. However, the size and location of the observed macro-porosity could not be such that it significantly diminishes the load capacity or the fatigue life of the cast part. Discarding such a part is economically unjustified. Our study focuses on the effect of different levels of porosity on the fatigue life and answering the question: when does the reduction in fatigue-life become statistically significant?

The effects of porosity on aluminium alloys have been researched before. Linder et al. [4] recorded a $15 \%$ drop in the material's strength when increasing the porosity from an initial 0.7 to $4.1 \%$. Wang [5] discovered that it is not only the percentage of porosity within the material, but also the size of the pores, that defines the effect on the fatigue-life.

When designing parts that experience dynamic loads, the fatigue strength of a material has to be known. It is usually determined by loading the specimens with dynamic loads at two different amplitude-load levels. Based on the scatter of the fatigue-life $N$ the durability curve representing a certain probability of rupture can be identified. When the materials' structure is not homogenous, the fatiguelife scatter is expected to increase. Fatigue-life tests can be stress or strain controlled. Loading a material containing inhomogeneities causes a localized increase in the stress and strain. Often, plastic deformation occurs around the inhomogeneities. Therefore, in cases where inhomogeneities are expected within the material, strain-controlled tests are preferred to ensure better control of a stress-strain state in the specimen. Considering that macro-pores were expected within the specimens, as well as a large scatter of the fatiguelife data, it was decided to perform the experiments at many different amplitude-strain levels. The results from this testing form a cloud of points in the $\varepsilon-N$ diagram. The results also indicated that despite the tests being strain-controlled the plastic component of 
the strain-life curve was not captured. Therefore, when performing statistical analyses, the same methods were applied as are used in stress-life fatigue-data analyses (see also Eq. (1)).

Since the literature is very scarce on this topic (no systematic survey of the statistical methods that could be applied for testing statistical differences between the fatigue-life curves was found) our goal was to find statistical methods that would first enable an identification of the fatigue-life reduction due to the level of porosity and, second, evaluate the statistical significance of the observed reduction. Three approaches were researched and compared to find the most suitable one for comparing different fatigue-life curves. The statistical significance was first estimated using a simple transformation of the fatigue-life data points $(\varepsilon, N)$ into a one-dimensional parameter combined with an analysis of the variance analysis (ANOVA). This parameter requires the assumption of equal slopes for all the fatigue-life curves. However, as it is shown later in the paper, that is not always the case. With the intent to avoid the assumption of equal slopes as well as the assumption of a linear relation between $\log \varepsilon$ and $\log N$ - a multivariate analysis of variance (MANOVA) for sets of non-transformed data points was employed. Finally, the estimation of a joint regression model with dummy variables for different groups of fatigue-life data combined with a significance test for the sets' dummy variables was carried out.

The theoretical background of the applied statistical methods is presented in Section 2. The experimental work is presented in Section 3, together with the used samples, the sample manufacturing and the testing plan. Three levels of porosity are defined, together with a non-destructive method for the detection of this porosity. The fatigue curves are presented at the end of this section. The results are presented in Section 4 and the concluding remarks are given in Section 5.

\section{THEORETICAL BACKGROUND}

\subsection{One-Dimensional Fatigue-Life Parameter Combined with the Univariate Analysis of Variance (ANOVA)}

$\varepsilon-N$ curves are usually characterized by the CoffinManson relationship [6] and [7]. However, due to the small amount of experimental data in our case it turned out that the plastic part of the Coffin-Manson curve was not well expressed. That is why we decided to apply only a linear $\varepsilon-N$ fatigue-life curve equation that should approximate a high-cycle fatigue regime.
By doing so we adopted a fatigue-life curve that is steeper than the elastic part, and more gradual than the plastic part, given by the Coffin-Manson relation. The relationship between the number-of-cycles-tofailure $N$ and amplitude strain level $\varepsilon$ can be written as follows:

$$
\frac{N_{1}}{N_{2}}=\left(\frac{\varepsilon_{1}}{\varepsilon_{2}}\right)^{-k} .
$$

From this equation it follows that:

$$
N_{1} \varepsilon_{2}^{-k}=N_{2} \varepsilon_{1}^{-k}=\text { const. }
$$

Therefore, a point with two coordinates ( $N$ and $\varepsilon$ ) can be transformed into a one-dimensional parameter using $N_{\mathrm{i}} \cdot \varepsilon_{\mathrm{j}}^{-k}$. This means that each of the measured data points should be reduced to a single value of this parameter if there was no scatter between the individual fatigue-life data points $(\varepsilon, N)$. Of course, because of the scattered fatigue-life data, the values of the one-dimensional parameter are also scattered.

If two fatigue-life curves are not identical, this parameter would be different for each of the two curves. As a result, the identity of two or more fatiguelife curves can be checked by testing the equality of the one-dimensional parameters from Eq. (2) for different data sets. However, this approach assumes the equality of the fatigue-life curve slopes $k$ for different groups of fatigue-life data. Therefore, when the slopes of the fatigue-life curves are not identical, the reduction to a one-dimensional parameter is made for the average parameter $\bar{k}$. Since the s fatigue-life data are reduced to a one-dimensional parameter, the significance between the different data sets is then tested using the one-way ANOVA test [8].

\subsection{Multivariate Analysis of Variance (MANOVA)}

MANOVA [9] is a multivariate extension of ANOVA. Its objective is to determine whether different groups of data are significantly different with respect to a given set of variables. The null and alternative hypotheses for multivariate statistical significance testing in MANOVA are for two groups of the bivariate variable $\mathbf{x}=\left(x_{1}, x_{2}\right)$ :

$$
H_{0}:\left(\begin{array}{l}
\mu_{11} \\
\mu_{21}
\end{array}\right)=\left(\begin{array}{l}
\mu_{12} \\
\mu_{22}
\end{array}\right) ; \quad H a:\left(\begin{array}{l}
\mu_{11} \\
\mu_{21}
\end{array}\right) \neq\left(\begin{array}{l}
\mu_{12} \\
\mu_{22}
\end{array}\right),
$$

where $\mu_{i j}$ is the mean of the $i^{\text {th }}$ variable for the $j^{\text {th }}$ group. The statistical distance between the mean vectors of the two groups is measured with the Mahalanobis distance (MD). The MD is given by [10]:

$$
M D_{\mathrm{ik}}=\left(\mathrm{x}_{\mathrm{i}}-\mathrm{x}_{\mathrm{k}}\right)^{\prime} S^{-1}\left(\mathrm{x}_{\mathrm{i}}-\mathrm{x}_{\mathrm{k}}\right) \text {, }
$$


where $\mathbf{x}$ is a $p \times 1$ vector of the coordinates and $\mathbf{S}$ is a $p \times p$ covariance matrix. The $\mathrm{MD}^{2}$ between the mean vectors is directly proportional to the differences between the two groups. The $\mathrm{MD}^{2}$ can then be transformed into various test statistics to determine whether it is large enough to claim that the difference between the groups is statistically significant. For multiple dependent variables the multivariate analogue for the differences between the groups is a function of the eigenvalue(s) $\lambda_{i}$ of the $\mathbf{S S C P}_{\mathrm{b}} \cdot \mathbf{S S C P}_{\mathrm{w}}{ }^{-1}$ matrix. Where $\mathbf{S S C P} \mathbf{P}_{\mathrm{b}}$ is between groups sum of squares and cross products matrix and $\mathbf{S S C P}_{\mathrm{w}}$ is within group sum of squares and cross products matrix. To test the null hypothesis against its alternative, eigenvalues $\lambda_{i}$ of the $\mathbf{S S C P}_{\mathrm{b}} \cdot \mathbf{S S C P}_{\mathrm{w}}{ }^{-1}$ matrix are combined together using, e.g., Pillai's trace $(V)$, Hotelling's trace $\left(T^{2}\right)$ or Wilks's lambda ( $\Lambda)$, Eq.(5) to (7), respectively.

$$
\begin{gathered}
V=\sum_{i=1}^{K} \frac{\lambda_{\mathrm{i}}}{1+\lambda_{\mathrm{i}}}, \\
T=\sum_{i=1}^{K} \lambda_{\mathrm{i}}, \\
\Lambda=\prod_{i=1}^{K} \frac{1}{1+\lambda_{\mathrm{i}}},
\end{gathered}
$$

where $\lambda_{i}$ is the eigenvalue for the $i^{\text {th }}$ discriminant variate and $K$ is the number of variates. In our case we have two dependent variables and two groups for the independent variable and therefore $K=1$. Because the $\mathrm{MD}$ is used to measure the distance between the group mean values and the MD is based on an elliptical distribution of the sample points around the group mean vector, the vectors $(\varepsilon, N)$ cannot be used directly, but must be transformed into their logarithms $(\log (\varepsilon)$, $\log (N))$.

Although the MANOVA reduces to a onedimensional parameter, it does have a significant advantage over the one-dimensional parameter introduced in Section 1.1, i.e., no transformation is carried out considering the slope of a regression line.

\subsection{Linear Regression with Dummy Variables}

Linear regression models the relationship between one dependent and one or more independent variables by fitting a linear equation to the observed data. The equation of the $\varepsilon-N$ fatigue-life curve, as defined in Section 1.1 (see Eq. (1)), becomes linear on a logarithmic scale. When generalized by $N_{1}=N$ and $\varepsilon_{1}$ $=\varepsilon$ Eq. (1) can be written as follows:

$$
\begin{aligned}
& \log (\varepsilon)=-\frac{1}{k} \log (N)+\left[\frac{1}{k} \log \left(N_{2}\right)+\log \left(\varepsilon_{2}\right)\right] . \\
& \text { If } b_{0}=k^{-1} \log \left(N_{2}\right)+\log \left(\varepsilon_{2}\right) \text { and } b_{1}=-k^{-1} \text { then }
\end{aligned}
$$

Eq. (8) can be written as:

$$
\log (\varepsilon)=b_{0}+b_{1} \cdot \log (N)
$$

Different specimen groups can be considered by introducing dummy variables $(D V)$ into Eq. (9). When analyzing the differences between two groups of data, one $D V$ is needed and Eq. (9) becomes:

$$
\begin{aligned}
\log (\varepsilon)_{\mathrm{i}}= & b_{0}+b_{1} \cdot \log (N)_{\mathrm{i}}+b_{2} \cdot D V_{\mathrm{i}}+ \\
& +b_{3} \cdot \log (N)_{\mathrm{i}} \cdot D V_{\mathrm{i}}, \\
D V & = \begin{cases}0 ; & \text { if reference group } \\
1 ; & \text { if other group }\end{cases}
\end{aligned}
$$

The regression coefficients $b_{0}$ and $b_{1}$ are the intercept and the slope, respectively, of a reference group. To account for the effect of the group, $b_{2} \cdot D V_{\mathrm{i}}$ is used to model the variation of the intercept between the groups and $b_{3} \cdot D V_{\mathrm{i}}$ is used to model the variation of the slope between the groups.

When analyzing three groups of data, two $D V \mathrm{~s}$ are needed and Eq. (9) becomes:

$$
\begin{aligned}
\log (\varepsilon)_{i} & =b_{0}+b_{1} \cdot \log (N)_{\mathrm{i}}+b_{2} \cdot D V_{\mathrm{i} 1}+b_{3} \cdot D V_{\mathrm{i} 2}+ \\
& +b_{4} \cdot \log (N)_{\mathrm{i}} \cdot D V_{\mathrm{i} 1}+b_{5} \cdot \log (N)_{\mathrm{i}} \cdot D V_{\mathrm{i} 2}, \\
D V_{1} & = \begin{cases}1 ; & \text { if group } 1 \\
0 ; & \text { if other group }\end{cases} \\
D V_{2} & = \begin{cases}1 ; & \text { if group } 2 \\
0 ; & \text { if other group }\end{cases}
\end{aligned}
$$

The regression coefficients $b_{0}$ and $b_{1}$ are the intercept and the slope, respectively, of a reference group. To account for the effect of the second group, $b_{2} \cdot D V_{i 1}$ is used to model the variation of the intercept and $b_{4} \cdot D V_{i 1}$ is used to model the variation of the slope between the second group and the reference group. Similarly, $b_{3} \cdot D V_{i 2}$ is used to model the variation of the intercept and $b_{5} \cdot D V_{i 2}$ is used to model the variation of the slope between the third group and the reference group.

\section{EXPERIMENTAL DATA}

Standard specimens according to the ASTM E606 [11] standard were manufactured from the AlSi9Cu3 alloy using pressure die casting - see Fig. 1. 


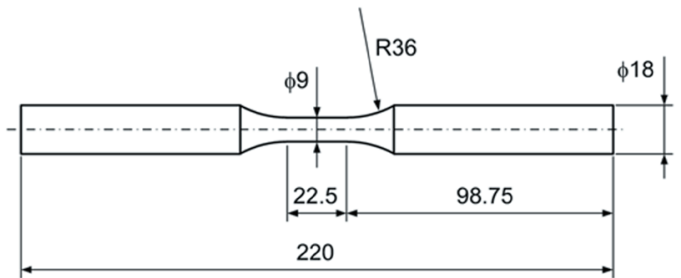

Fig. 1. Shape and dimensions of standard specimen

In order to study the effect of different porosity levels on the fatigue life the porosity had to be introduced into the specimens. This was achieved by not applying the usual pressure of 710 bar in the third phase of casting, used to condense the aluminium. A total of 33 specimens were manufactured. The condition of the specimen's interior was unknown after the manufacturing process. To determine the level of porosity induced in each specimen, the samples were X-rayed in two perpendicular planes. Based on the acquired x-ray images, three levels of porosity were defined:

- level A: no macro-pores are visible in the narrow part of the specimen, Fig. 2a,

- level B: in the narrow part of the specimen a small number of macro-pores (usually only one) are detected, Fig. 2b,

- level C: in the narrow part of the specimen a large number of macro-pores are detected, Fig. 2c.

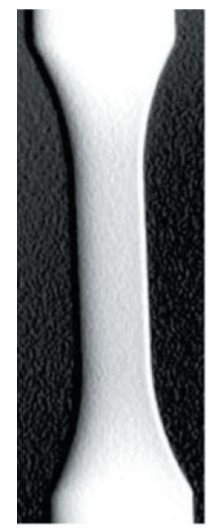

(a)

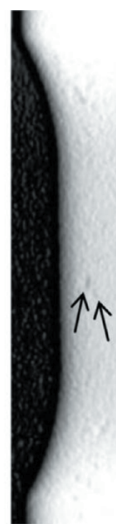

(b)

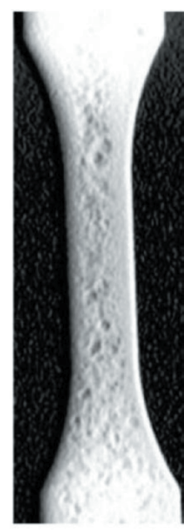

(c)
Fig. 2. X-ray images of samples; a) porosity level $A$, b) porosity level $B$ (macro-pores are indicated with arrows) and, c) porosity level $\mathrm{C}$

Out of the 33 samples examined, 15 were characterized with the porosity level A, 10 were characterized with the porosity level $\mathrm{B}$ and 8 were characterized with the porosity level C. In addition, another series of AlSi9Cu3 fatigue-life data from our previous research [12] was included in the study.
These data correspond to homogenous specimens and represent the reference data from another production series of the specimens. These data were marked with MJ, after the first author of the previous research. To define the amplitude-strain levels for the fatigue-life experiments, destructive tensile tests were performed for one specimen of each porosity level. The tensiletest results were compared to the results of the homogenous specimens of AlSi9Cu3 from [12]. The results are listed in Table 1 and shown in Fig. 3.

Table 1. Material parameters of porous and homogenous AISigCu3

\begin{tabular}{ccccc}
\hline $\begin{array}{c}\text { Por. } \\
\text { level }\end{array}$ & $\begin{array}{c}E \\
{[\mathrm{MPa}]}\end{array}$ & $\begin{array}{c}R_{p 02} \\
{[\mathrm{MPa}]}\end{array}$ & $\begin{array}{c}R_{m} \\
{[\mathrm{MPa}]}\end{array}$ & $\begin{array}{c}\varepsilon_{\text {rup }} \\
{[\%]}\end{array}$ \\
\hline $\mathrm{A}$ & 79631 & 178 & 323 & 4.35 \\
\hline $\mathrm{B}$ & 70777 & 154 & 271 & 2.31 \\
\hline $\mathrm{C}$ & 69143 & 152 & 248 & 2.48 \\
\hline $\mathrm{MJ} \# 1$ & 80212 & 164 & 304 & 4.93 \\
\hline $\mathrm{MJ} \# 2$ & 74761 & 137 & 312 & 5.21 \\
\hline $\mathrm{MJ} \# 3$ & 78649 & 136 & 303 & 4.85 \\
\hline
\end{tabular}

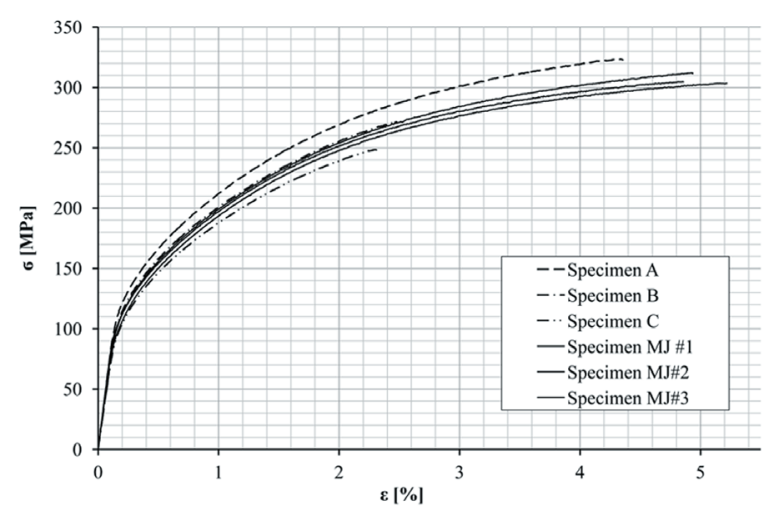

Fig. 3. Tensile tests of porous specimens and homogenous specimens

When the material's structure is not homogenous, the fatigue-life scatter is expected to increase compared to the homogenous structure [2]. Therefore, the specimens were loaded at many different amplitude-strain levels. The dynamic tests were fully reversal, strain controlled, with the amplitude-strain levels between 0.1 and $0.4 \%$. All the fatigue-life experiments were performed on a MTS 810.22 servohydraulic test stand at the company CIMOS, Slovenia. The strain was measured using an MTS 632.53F-14 extensometer with a measuring distance of $12 \mathrm{~mm}$. The tests were performed under atmospheric pressure and at a temperature of $21^{\circ} \mathrm{C}$.

The experimental fatigue-life data for the four groups (MJ, A, B and C) are presented in Fig. 4. In the same figure the $\varepsilon-N$ curves modelled by Eq. (1) and representing 10,50 and $90 \%$ probability of rupture 
are also shown. The scatter of the $\varepsilon-N$ curves was modelled using a Weibull probability density function:

$$
f(N)=\frac{\beta}{\eta}\left(\frac{N}{\eta}\right)^{\beta-1} \cdot e^{\left\{-\left(\frac{N}{\eta}\right)^{\beta}\right\}} .
$$

In Eq. (12) the scale factor $\eta$ is dependent on the amplitude-strain level via the Basquin equation:

$$
\eta=\eta(\varepsilon)=10^{c_{0}+c_{1} \cdot \log (\varepsilon)} ; \quad \mathrm{c}_{0}>0, \quad c_{1}<0,
$$

where:

$$
c_{0}=\log \left(N_{2}\right)+k \cdot \log \left(\varepsilon_{2}\right), \quad c_{1}=-k .
$$

and the shape factor $\beta$ is constant. The parameters $c_{0}$, $c_{1}$ and $\beta$ were determined according to the procedure that was derived by Klemenc and Fajdiga [13].

From the diagram in Fig. 4 we can see that the fatigue curves differ from each other with respect to the level of porosity. The specimens with porosity levels $\mathrm{B}$ and $\mathrm{C}$ display a much greater scatter than the specimens from the groups with level A and MJ. When observing a certain strain level we find that the specimens $\mathrm{C}$ have the shortest fatigue life, followed by the specimens B, and the specimens A, which have the longest fatigue life. There is also a slight discrepancy between the specimens A and the specimens MJ. This suggests that the production series influences the fatigue life to a certain degree.

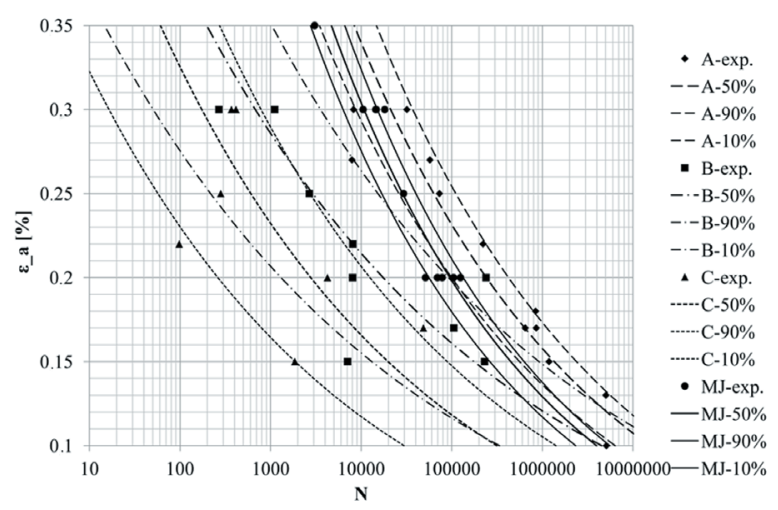

Fig. 4. Fatigue curves for $A, B$ and $C$ levels of porosity and homogenous specimens $\mathrm{MJ}$

\section{RESULTS AND DISCUSSION}

We had a relatively small number of specimens and the specimen groups were of unequal size (14 samples with level-A porosity, 9 samples with level-B porosity, 7 samples with level-C porosity and an additional 16 unaltered MJ samples). This can affect the outcome of the statistical analysis and can lead to a false conclusion regarding the statistical significance of the differences in the fatigue life. To assess the influence of the different sample-set sizes on the statistical significance, additional fatigue-life data were generated in accordance with the $\varepsilon-N$ curve models from Fig. 4. The data for each specimen group were generated using a Weibull random generator with its parameters set-up according to Table 2 for the amplitude-strain levels between 0.1 and $0.4 \%$. First, the number of sample points in each group was levelled to match the largest sample set (16 sample points). Second, to study the effect of the sample-set size on the statistical significance, additional sample points were generated so that the sample-set sizes were increased to 40 and 80 samples. The experimentally obtained and simulated data points are shown in Fig. 5. A regression equation for each data set is displayed on the presented plots. The slope of the regression line and the parameter $c_{1}$ are correlated with the following relation $c_{1}=-1 /$ slope. In the following subsections the significance of the fatigue-life reduction due to the macro porosity is analysed.

Table 2. Parameters of $A, B, C$ and $M J$ fatigue curves

\begin{tabular}{cccc}
\hline Por. level & $C_{0}$ & $C_{1}$ & $\beta$ \\
\hline A & 1.2622 & -6.0001 & 2.1329 \\
\hline B & -1.1397 & -8.0143 & 0.7252 \\
\hline C & -1.1281 & -6.8248 & 0.7980 \\
\hline MJ & 1.2630 & -5.3798 & 3.5454 \\
\hline
\end{tabular}

\subsection{ANOVA for the One-Dimensional Fatigue-Life Parameter}

The experimental results with two dimensions $(\varepsilon, N)$ were transformed into a one-dimensional parameter, as described in Section 2.1, see Fig. 6. The four groups of data were then compared using a one-way ANOVA, with the results being presented in Table 3 .

Table 3. ANOVA results for one-dimensional parameter with the number of different porous specimens in each sample

\begin{tabular}{ccc}
\hline Sample & $F$-stat. & $p$-val. \\
\hline $14 \mathrm{~A}+10 \mathrm{~B}+7 \mathrm{C}+16 \mathrm{MJ}$ & 13.743 & 0.000 \\
\hline $16 \mathrm{~A}+16 \mathrm{~B}+16 \mathrm{C}+16 \mathrm{MJ}$ & 18.151 & 0.000 \\
\hline $40 \mathrm{~A}+40 \mathrm{~B}+40 \mathrm{C}+40 \mathrm{MJ}$ & 25.914 & 0.000 \\
\hline $80 \mathrm{~A}+80 \mathrm{~B}+80 \mathrm{C}+80 \mathrm{MJ}$ & 48.877 & 0.000 \\
\hline
\end{tabular}

The analyses of the experimental samples showed that the differences between the original groups of the fatigue-life data are statistically significant $(F=$ 13.743 and $p<0.05$ ); where the $\mathrm{p}$-value is defined as the smallest significance level at which the null hypothesis would be rejected [8]. By increasing the number of samples in each set, the differences 


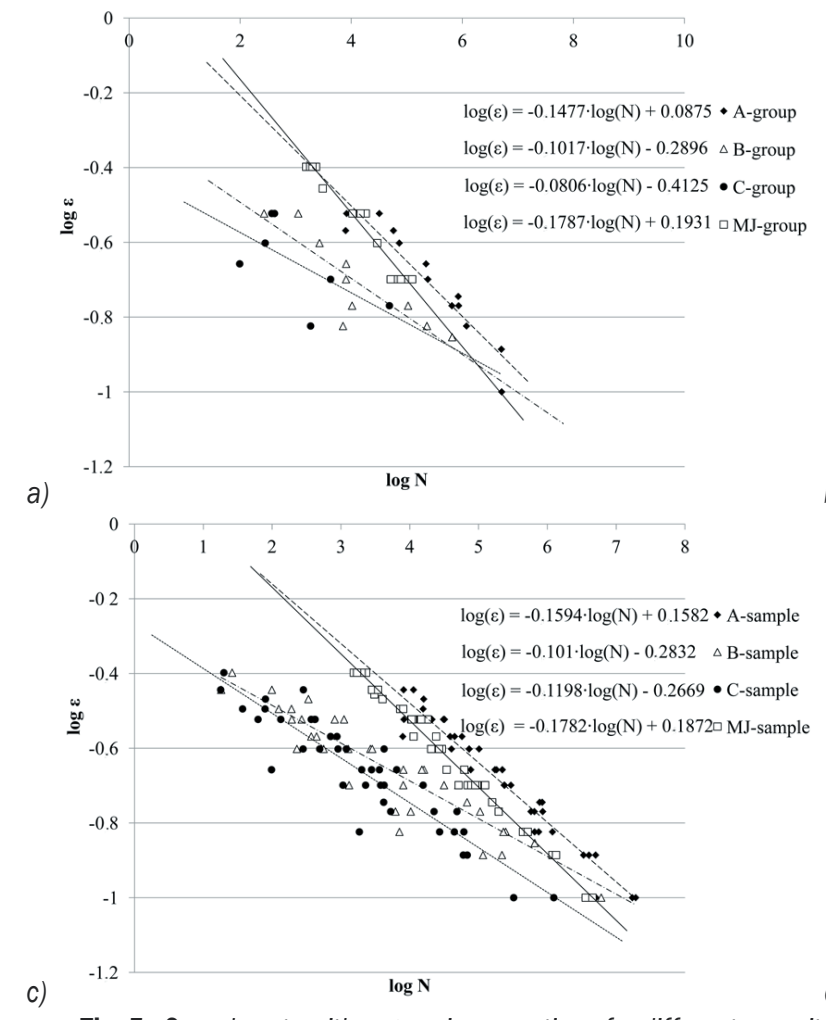

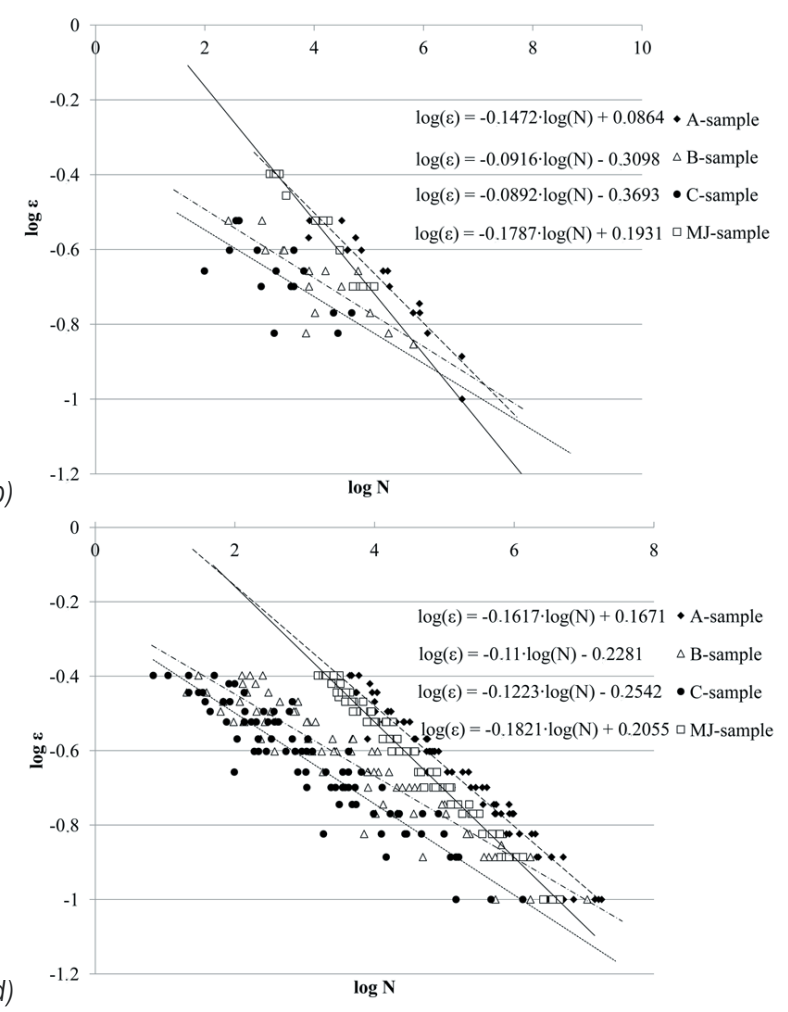

d)
Fig. 5. Sample sets with regression equations for different porosity levels; a) experimental samples, b) 16, c) 40, and d) 80 samples between the groups remain significant $(p<0.05)$. When comparing the results from the sample sets with 40 samples and the sample sets with 80 samples, the tests become even more significant, implying that there is no justification for increasing the size of the sample sets beyond 40 samples per set.

While this approach manages to distinguish between the groups, one should keep in mind that the slopes are assumed to be equal within the statistical scatter. From Fig. 5 it is clear that the slopes do vary between the groups and therefore the results of the ANOVA analysis are unreliable in our case.

\subsection{Multivariate Analysis of Variance}

The analyses were carried out by always comparing two groups of the fatigue-life data at the same time: groups $\mathrm{A}$ and $\mathrm{MJ}$, groups $\mathrm{A}$ and $\mathrm{B}$, groups $\mathrm{A}$ and $\mathrm{C}$ and groups $\mathrm{B}$ and $\mathrm{C}$. The results are given in Tables 4 to 7 , respectively.

The discussion of the results is divided into two parts: first, the analyses involving group A are addressed and, second, the analyses of groups B and $\mathrm{C}$ are addressed. When comparing group $\mathrm{A}$ with the other groups, the power of the multivariate tests is large (power $\approx 1.00$ ), suggesting that the probability of rejecting the null hypothesis (see Eq. 3) when it is false is very low. Regardless of the size of the sample set the multivariate test statistics (Pillai's trace, Wilks's lambda and Hotelling's trace) indicate a statistically significant effect for the level of porosity at $p<0.05$. The higher the porosity level, the greater is the effect on the fatigue life. But there can be more than one reason for a statistical significance found by a multivariate test, for example, the data could be shifted along the centre line or they could be shifted vertically downwards or upwards, to the left or to the

Table 4. MANOVA results for groups $A$ and $M J$

\begin{tabular}{|c|c|c|c|c|c|}
\hline & & $14-16$ & $16-16$ & $40-40$ & $80-80$ \\
\hline \multirow{2}{*}{$V$} & Val. & 0.475 & 0.469 & 0.548 & 0.544 \\
\hline & $p$ & 0.000 & 0.000 & 0.000 & 0.000 \\
\hline \multirow{2}{*}{$T^{2}$} & Val. & 0.905 & 0.886 & 1.212 & 1.195 \\
\hline & $p$ & 0.000 & 0.000 & 0.000 & 0.000 \\
\hline \multirow{2}{*}{$\Lambda$} & Val. & 0.524 & 0.530 & 0.451 & 0.455 \\
\hline & $p$ & 0.000 & 0.000 & 0.000 & 0.000 \\
\hline \multicolumn{2}{|c|}{ Power } & 0.99 & 0.99 & 0.99 & 1.00 \\
\hline \multirow{4}{*}{$\begin{array}{l}z \\
\text { D } \\
\omega \\
\tilde{O} \\
\underline{0}\end{array}$} & $p$ & 0.000 & 0.000 & 0.001 & 0.001 \\
\hline & Power & 0.984 & 0.987 & 0.919 & 0.923 \\
\hline & $p$ & 0.002 & 0.002 & 0.098 & 0.248 \\
\hline & Power & 0.894 & 0.896 & 0.379 & 0.207 \\
\hline
\end{tabular}



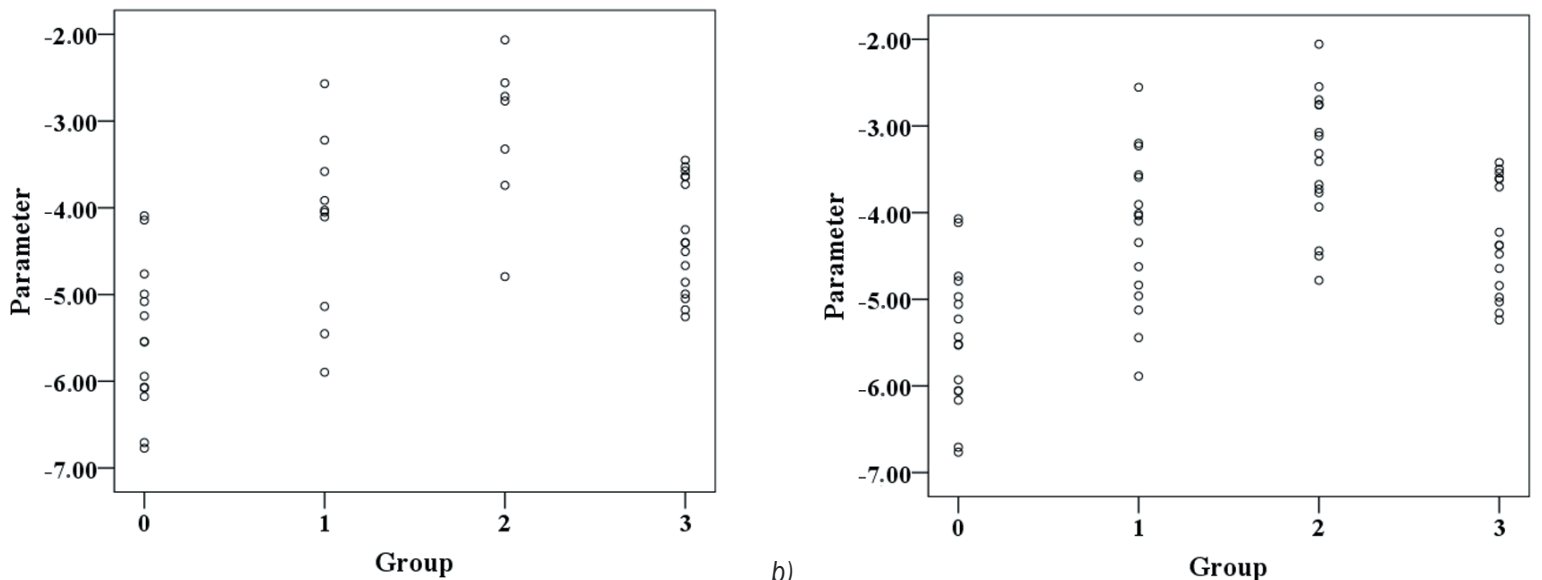

a)

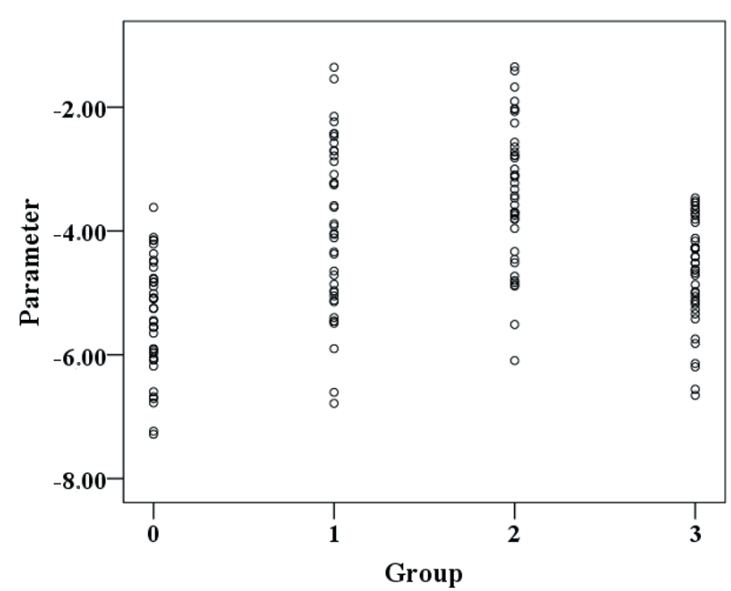

b)

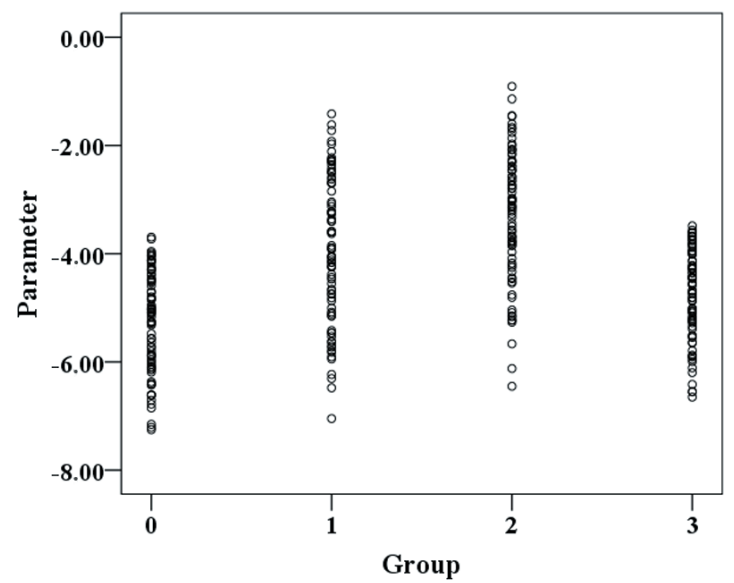

Fig. 6. Graphical presentation of one-dimensional parameter $N \cdot \varepsilon^{-k}$ for; a) experimental sample set, b) 16 samples in set, c) 40 samples in set and d) 80 samples in set; group $A=(0)$, group $B=(1)$, group $C=(2)$, group $M J=(3)$

right. After each multivariate test a univariate test was performed to determine which variable contributes to the differences between the two groups. The univariate tests indicate that $\log (N)$ contributes to the differences between the groups, regardless of which group is being compared to group A and regardless of the sample-set size. The univariate tests yield a different result for $\log (\varepsilon)$. The only case where it reaches a significance level is in the analysis of groups A and MJ, when the number of samples was no more than 16 samples per set. The reason for this can be found in Fig. 6. Note that the tests for specimens A and specimens MJ were not performed in the same strain range; therefore, the univariate tests are significant with respect to $\log (\varepsilon)$. When the number of specimens is increased and the data is more equally distributed across the strain range, the test becomes significant. A similar reason explains why $\log (\varepsilon)$ is not significant when comparing group A to group B, and group A to group C. Despite all the MANOVA tests involving the group A being significant, we can see from Table 3 that the ANOVA test for the variable $\log (N)$ and an increased number of data points is not significant at the $0.1 \%$ significance level, which implies that the difference is not due to the porosity, but due to the production series.

Table 5. MANOVA results for groups $A$ and $B$

\begin{tabular}{|c|c|c|c|c|c|}
\hline & & $14-10$ & $16-16$ & $40-40$ & $80-80$ \\
\hline \multirow{2}{*}{$V$} & Val. & 0.713 & 0.653 & 0.704 & 0.701 \\
\hline & $p$ & 0.000 & 0.000 & 0.000 & 0.000 \\
\hline \multirow{2}{*}{$T^{2}$} & Val. & 2.485 & 1.888 & 2.380 & 2.347 \\
\hline & $p$ & 0.000 & 0.000 & 0.000 & 0.000 \\
\hline \multirow{2}{*}{$\Lambda$} & Val. & 0.286 & 0.346 & 0.295 & 0.298 \\
\hline & $p$ & 0.000 & 0.000 & 0.000 & 0.000 \\
\hline \multicolumn{2}{|c|}{ Power } & 0.99 & 0.99 & 0.99 & 1.00 \\
\hline \multirow{4}{*}{ 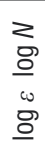 } & $p$ & 0.004 & 0.001 & 0.000 & 0.000 \\
\hline & Power & 0.859 & 0.960 & 0.999 & 1.00 \\
\hline & $p$ & 0.971 & 0.847 & 0.925 & 0.950 \\
\hline & Power & 0.040 & 0.044 & 0.035 & 0.032 \\
\hline
\end{tabular}

When discussing the analysis results of groups B and $\mathrm{C}$ it is clear that all the test statistics show a non- 
significant difference between the groups ( $p=0.081$ ). The univariate tests indicate that none of the means are significantly different for the two groups. However, a level of statistical significance $(p<0.05)$ is reached as soon as the sample sets are made equal and the number of samples in each set is increased to 16 . At this point $\log (N)$ becomes significant, while $\log (\varepsilon)$, like in the other analyses, never does. Nevertheless, it can be concluded that the statistical difference in the fatigue-life data is the smallest between the groups representing level-B and level-C porosity.

Table 6. MANOVA results for groups $A$ and $C$

\begin{tabular}{|c|c|c|c|c|c|}
\hline & & $14-7$ & 16-16 & $40-40$ & $80-80$ \\
\hline \multirow{2}{*}{$V$} & Val. & 0.824 & 0.818 & 0.869 & 0.878 \\
\hline & $p$ & 0.000 & 0.000 & 0.000 & 0.000 \\
\hline \multirow{2}{*}{$T^{2}$} & Val. & 4.684 & 4.500 & 6.634 & 7.212 \\
\hline & $p$ & 0.000 & 0.000 & 0.000 & 0.000 \\
\hline \multirow{2}{*}{$\Lambda$} & Val. & 0.175 & 0.181 & 0.130 & 0.121 \\
\hline & $p$ & 0.000 & 0.000 & 0.000 & 0.000 \\
\hline \multicolumn{2}{|c|}{ Power } & 0.99 & 0.99 & 0.99 & 1.00 \\
\hline \multirow{4}{*}{$\begin{array}{l}z \\
\text { D } \\
\omega \\
\text { Do }\end{array}$} & $p$ & 0.000 & 0.000 & 0.000 & 0.000 \\
\hline & Power & 0.999 & 1.000 & 1.000 & 1.000 \\
\hline & $p$ & 0.471 & 0.494 & 0.740 & 0.824 \\
\hline & Power & 0.127 & 0.107 & 0.049 & 0.040 \\
\hline
\end{tabular}

Table 7. MANOVA results for groups $B$ and $C$

\begin{tabular}{|c|c|c|c|c|c|}
\hline & & 14-7 & 16-16 & $40-40$ & $80-80$ \\
\hline \multirow{2}{*}{$V$} & Val. & 0.301 & 0.281 & 0.182 & 0.301 \\
\hline & $p$ & 0.081 & 0.008 & 0.000 & 0.000 \\
\hline \multirow{2}{*}{$T^{2}$} & Val. & 0.431 & 0.392 & 0.222 & 0.432 \\
\hline & $p$ & 0.081 & 0.008 & 0.000 & 0.000 \\
\hline \multirow{2}{*}{$\Lambda$} & Val. & 0.698 & 0.718 & 0.817 & 0.698 \\
\hline & $p$ & 0.081 & 0.008 & 0.000 & 0.000 \\
\hline \multicolumn{2}{|c|}{ Power } & 0.99 & 0.99 & 0.49 & 0.82 \\
\hline \multirow{4}{*}{$\begin{array}{l}z \\
\text { D } \\
\omega \\
\text { OD } \\
\text { D }\end{array}$} & $p$ & 0.49 & 0.013 & 0.066 & 0.003 \\
\hline & Power & 0.515 & 0.725 & 0.453 & 0.854 \\
\hline & $p$ & 0.436 & 0.567 & 0.806 & 0.871 \\
\hline & Power & 0.142 & 0.060 & 0.439 & 0.036 \\
\hline
\end{tabular}

Using MANOVA we can detect a multivariate significance even in the cases where no univariate significance is detected. The MANOVA weaknesses are displayed in Table 4 when observing the univariate results for $\log (\varepsilon)$ : it is clear that the MANOVA would fail to identify data belonging to the same fatigue curve if the tests were carried out at different strain levels.

\subsection{Linear Regression Using Dummy Variables}

Altogether, four regression models with dummy variables were built: the first comparing groups $\mathrm{A}$ and $\mathrm{MJ}$, the second comparing groups $\mathrm{A}, \mathrm{B}$ and $\mathrm{C}$ and the third comparing groups $\mathrm{B}$ and $\mathrm{C}$. Based on the considered number of fatigue-life data groups in the individual regression models, the number of required dummy variables was defined. The dummyvariable coding system is given in Table 8 . The linearregression coefficient together with their significances are presented in Tables 9 to 12 .

Table 8. Indicator variable coding system for performed analysis

\begin{tabular}{cccc}
\hline Model & Group & DV1 & DV2 \\
\hline \multirow{2}{*}{ A - MJ } & A & 0 & $/$ \\
\cline { 2 - 4 } & MJ & 1 & $/$ \\
\hline \multirow{3}{*}{ A - B - C } & A & 0 & 0 \\
\cline { 2 - 4 } & B & 1 & 0 \\
\hline \multirow{2yyy}{*}{ B - C } & C & 0 & 1 \\
\cline { 2 - 4 } & B & 0 & $/$ \\
\hline \multirow{2}{*}{ A - BC } & C & 1 & $/$ \\
\cline { 2 - 4 } & BC & 0 & $/$ \\
\hline
\end{tabular}

Table 9. Regression with indicator coding results for groups $A$ and MJ

\begin{tabular}{cccc}
\hline Sample & & $\mathrm{b}_{\mathrm{i}}$ & $p$-value \\
\hline & $\mathrm{b}_{0}$ & 0.088 & 0.180 \\
\cline { 2 - 4 } $14 \mathrm{~A}+16 \mathrm{MJ}$ & $\mathrm{b}_{1}$ & -0.148 & 0.000 \\
\cline { 2 - 4 } $\mathrm{R}^{2}{ }_{\mathrm{adj}}=0.939$ & $\mathrm{~b}_{2}$ & 0.106 & 0.234 \\
\cline { 2 - 4 } & $\mathrm{b}_{3}$ & -0.031 & 0.104 \\
\hline & $\mathrm{b}_{0}$ & 0.086 & 0.161 \\
\cline { 2 - 4 } $16 \mathrm{~A}+16 \mathrm{MJ}$ & $\mathrm{b}_{1}$ & -0.147 & 0.000 \\
\cline { 2 - 4 } $\mathrm{R}_{\mathrm{adj}}=0.938$ & $\mathrm{~b}_{2}$ & 0.107 & 0.210 \\
\cline { 2 - 4 } & $\mathrm{b}_{3}$ & -0.032 & 0.088 \\
\hline & $\mathrm{b}_{0}$ & 0.158 & 0.000 \\
\cline { 2 - 4 } $40 \mathrm{~A}+40 \mathrm{MJ}$ & $\mathrm{b}_{1}$ & -0.159 & 0.000 \\
\cline { 2 - 4 } $\mathrm{R}^{2}{ }_{\mathrm{adj}}=0.969$ & $\mathrm{~b}_{2}$ & 0.029 & 0.399 \\
\cline { 2 - 4 } & $\mathrm{b}_{3}$ & -0.019 & 0.008 \\
\hline & $\mathrm{b}_{0}$ & 0.167 & 0.000 \\
\cline { 2 - 4 } $80 \mathrm{~A}+80 \mathrm{MJ}$ & $\mathrm{b}_{1}$ & -0.162 & 0.000 \\
\cline { 2 - 4 } $\mathrm{R}{ }_{\mathrm{adj}}=0.976$ & $\mathrm{~b}_{2}$ & 0.038 & 0.070 \\
\cline { 2 - 4 } & $\mathrm{b}_{3}$ & -0.020 & 0.000 \\
\hline
\end{tabular}

The analysis with group A and group MJ was carried out with the intension to investigate the effect of the production series on the fatigue life. Observing the results (see Table 9) we see that the adjusted $R^{2}$ values are very high $\left(R^{2}>0.93\right)$; where the $\mathrm{R}^{2}$ coefficient is defined as the squared correlation of the dependent variable and the fitted values. [9] Experimental sample sets and sets with 16 samples are not significantly different with respect to all the model parameters (intercept and slope). The fact that neither the slope nor the intercept differ significantly at the 5\% significance level between the two groups indicates 
that micro-porosity does not have a significant impact on the fatigue life. Increasing the sample-set size to 40 samples and above the significance of the slope differences increases. Even when the set size is increased to 80 the intercepts are not significantly different at the $5 \%$ significance level.

Once we established that the production series does not have a significant effect on the fatigue life, an analysis was conducted to see whether macro-porosity has a significant effect on the fatigue life. Groups $\mathrm{B}$ and $\mathrm{C}$ were compared to the reference group $\mathrm{A}$. Observing the results of the analysis (Table 10) we see that in the experimental data and the 16 sample data sets the intercepts of groups B and C are significantly different from that of group A $(p<0.05)$. The slopes, however, are not significantly different $(p>0.05)$. When the number of samples per set is increased to 40 the slopes as well as the intercepts of groups B and C differ significantly from group A. From these results it is possible to conclude that the macro-porosity in the critical cross-section has a significant effect on the fatigue life. The results also suggest that there is no point in increasing the sample-set size beyond 40 .

Table 10. Regression with indicator coding results for groups $A, B$ and $\mathrm{C}$

\begin{tabular}{|c|c|c|c|}
\hline Sample & & $b_{i}$ & $p$-value \\
\hline \multirow{6}{*}{$\begin{array}{c}14 \mathrm{~A}+10 \mathrm{~B}+7 \mathrm{C} \\
\mathrm{R}^{2}{ }_{\mathrm{adj}}=0.722\end{array}$} & $\mathrm{~b}_{0}$ & 0.088 & 0.442 \\
\hline & $b_{1}$ & -0.148 & 0.000 \\
\hline & $b_{2}$ & -0.377 & 0.015 \\
\hline & $b_{3}$ & -0.500 & 0.002 \\
\hline & $b_{4}$ & 0.046 & 0.136 \\
\hline & $b_{5}$ & 0.067 & 0.080 \\
\hline \multirow{6}{*}{$\begin{array}{c}16 A+16 B+16 C \\
R^{2}{ }_{a d j}=0.691\end{array}$} & $\mathrm{~b}_{0}$ & 0.086 & 0.391 \\
\hline & $b_{1}$ & -0.147 & 0.000 \\
\hline & $b_{2}$ & -0.396 & 0.003 \\
\hline & $b_{3}$ & -0.456 & 0.001 \\
\hline & $b_{4}$ & 0.056 & 0.036 \\
\hline & $b_{5}$ & 0.058 & 0.043 \\
\hline \multirow{6}{*}{$\begin{array}{c}40 A+40 B+40 C \\
R^{2}{ }_{a d j}=0.882\end{array}$} & $\mathrm{~b}_{0}$ & 0.158 & 0.001 \\
\hline & $b_{1}$ & -0.159 & 0.000 \\
\hline & $b_{2}$ & -0.441 & 0.000 \\
\hline & $b_{3}$ & -0.425 & 0.000 \\
\hline & $b_{4}$ & 0.058 & 0.000 \\
\hline & $b_{5}$ & 0.040 & 0.001 \\
\hline \multirow{6}{*}{$\begin{array}{c}80 A+80 B+80 C \\
R^{2}{ }_{a d j}=0.908\end{array}$} & $\mathrm{~b}_{0}$ & 0.167 & 0.000 \\
\hline & $b_{1}$ & -0.162 & 0.000 \\
\hline & $b_{2}$ & -0.395 & 0.000 \\
\hline & $b_{3}$ & -0.421 & 0.000 \\
\hline & $b_{4}$ & 0.052 & 0.000 \\
\hline & $b_{5}$ & 0.039 & 0.000 \\
\hline
\end{tabular}

Once the significance in the differences between groups $\mathrm{A}, \mathrm{B}$ and $\mathrm{C}$ was identified, groups $\mathrm{B}$ and $\mathrm{C}$ were compared in a separate analysis (see Table 11). The experimental samples show no significant difference between the two groups in terms of slope or intercept $(p>0.05)$. However, increasing the sample size to 40 does increase the adjusted $R^{2}$ value. Neither the slope nor the intercept reach the level of significance $(p>$ $0.05)$.

After failing to find a significant difference between the levels of porosity $\mathrm{B}$ and $\mathrm{C}$ we conducted an additional analysis in which we joined the samples with porosity levels $\mathrm{B}$ and $\mathrm{C}$ to form a new group named BC. Group BC contains all the specimens with macro-pores within the structure. Only the experimental samples were analyzed (see Table 12). The results show that the intercept as well as the slope differ significantly between the groups $(p<0.05)$. The adjusted $R^{2}$ value $\left(R^{2}=0.721\right.$, Table 12$)$ is roughly the same as in the analysis with three porosity levels $\left(R^{2}\right.$ $=0.722$, Table 9).

Table 11. Regression with indicator coding results for groups $B$ and $\mathrm{C}$

\begin{tabular}{cccc}
\hline Sample & & $\mathrm{b}_{\mathrm{i}}$ & $p$-value \\
\hline & $\mathrm{b}_{0}$ & -0.290 & 0.019 \\
\cline { 2 - 4 } $14 \mathrm{~A}+16 \mathrm{MJ}$ & $\mathrm{b}_{1}$ & -0.102 & 0.002 \\
\cline { 2 - 4 } $\mathrm{R}^{2}{ }_{\mathrm{adj}}=0.939$ & $\mathrm{~b}_{2}$ & -0.123 & 0.448 \\
\cline { 2 - 4 } & $\mathrm{b}_{3}$ & 0.021 & 0.643 \\
\hline & $\mathrm{b}_{0}$ & -0.310 & 0.001 \\
\cline { 2 - 4 } $16 \mathrm{~A}+16 \mathrm{MJ}$ & $\mathrm{b}_{1}$ & -0.092 & 0.000 \\
\cline { 2 - 4 } $\mathrm{R}^{2}{ }_{\mathrm{adj}}=0.938$ & $\mathrm{~b}_{2}$ & -0.059 & 0.602 \\
\cline { 2 - 4 } & $\mathrm{b}_{3}$ & 0.002 & 0.938 \\
\hline & $\mathrm{b}_{0}$ & -0.283 & 0.000 \\
\cline { 2 - 4 } $40 \mathrm{~A}+40 \mathrm{MJ}$ & $\mathrm{b}_{1}$ & -0.101 & 0.000 \\
\cline { 2 - 4 } $\mathrm{R}^{2}{ }_{\mathrm{adj}}=0.969$ & $\mathrm{~b}_{2}$ & 0.016 & 0.683 \\
\cline { 2 - 4 } & $\mathrm{b}_{3}$ & -0.019 & 0.083 \\
\hline & $\mathrm{b}_{0}$ & -0.228 & 0.000 \\
\cline { 2 - 4 } $80 \mathrm{~A}+80 \mathrm{MJ}$ & $\mathrm{b}_{1}$ & -0.110 & 0.000 \\
\cline { 2 - 4 } $\mathrm{R}^{2}{ }_{\mathrm{adj}}=0.976$ & $\mathrm{~b}_{2}$ & -0.026 & 0.307 \\
\cline { 2 - 4 } & $\mathrm{b}_{3}$ & -0.012 & 0.076 \\
\hline
\end{tabular}

Table 12. Regression with indicator coding results for groups $A$ and joined groups BC

\begin{tabular}{cccc}
\hline Sample & & $\mathrm{b}_{\mathrm{i}}$ & $p$-value \\
\hline & $\mathrm{b}_{0}$ & 0.088 & 0.443 \\
\cline { 2 - 4 } $14 \mathrm{~A}+17 \mathrm{BC}$ & $\mathrm{b}_{1}$ & -0.148 & 0.000 \\
\cline { 2 - 4 } $\mathrm{R}^{2}{ }_{\mathrm{adj}}=0.721$ & $\mathrm{~b}_{2}$ & -0.469 & 0.001 \\
\cline { 2 - 4 } & $\mathrm{b}_{3}$ & 0.064 & 0.019 \\
\hline
\end{tabular}

\section{CONCLUSIONS}

The study showed that macro-porosity at a critical specimen cross-section affects the fatigue life of an AlSi9Cu3 aluminium alloy. Among the three defined 
levels of porosity, the level without macro-porosity was proven to have the least impact on fatigue. However, if larger pores are present within the critical cross-section of the sample, the fatigue life is drastically reduced.

The performed statistical analysis using three different methods provides guidelines for selecting the proper approach for large-scatter fatigue-data analysis. Despite reducing to a single-dimensional value the MANOVA proved to have a major advantage over the one-dimensional fatigue parameter combined with the one-way ANOVA. The MANOVA does not include a transformation where the slope would be regarded; therefore, allowing the researcher to compare fatigue curves with various slopes. The downside is that the MANOVA is not able to identify sample sets belonging to the same fatigue curve if the fatigue-life data in different data sets are shifted along the fatiguelife curve in the direction of the amplitude-strain levels. Linear regression with indicator variables proved to be the most informative approach, offering information about the significance of the differences between groups as well as the sources of the observed differences. Based on the results it is possible to identify data belonging to the same fatigue curve, even if the data are acquired for different strain ranges.

Our analysis also provides some guidelines for planning an experiment to investigate the fatigue life of inhomogeneous materials. A large amount of experimental data are always desired; however, for large sample sizes even small differences between different sample sets become statistically significant. When proving that even production series differ between each other, it would also be useful to assess the practical significance of the differences between groups. For that reason we suggest no more than 40 samples per data set in order to obtain relevant results regarding the significance of the differences between the groups.

The results of this study clearly show that specimens without macro-porosity do not exhibit a major change in the fatigue life between different production series if the processing parameters are kept constant. On the other hand, the presence of macro-porosity within the critical cross-section of the specimen has a detrimental effect on the fatigue life and reduces it drastically, regardless of the pore distribution. One of the most significant added values of our research was that we have calculated the magnitude and the statistical significance of the fatigue-life reduction due to the macro-porosity. This is a very favourable result for a practical application, since macro-porosity can be estimated beforehand using numerical simulations and by knowing its effect we can estimate whether the predicted macro-porosity for different cross-sections of the product would result in a significant fatigue-life reduction for that product.

\section{REFERENCES}

[1] Skallerund, B., Iveland, T., Harkegard, G. (1993). Fatigue life assessment of aluminum cast alloys. Part $I$. Engineering Fracture Mechanics, vol. 44, p. 857-874, DOI:10.1016/0013-7944(93)90108-5.

[2] Murakami, Y., Endo, M. (1994). Effects of defects, inclusions and inhomogeneities on fatigue strength. International Journal of Fatigue, vol. 16, p. 163-182, DOI:10.1016/0142-1123(94)90001-9.

[3] Gwiżdż, A., Pysz, S., Dworak, P. (2010). MAGMAsoft simulations applied in verification of technology to produce new range of alloy steel castings. Archives of Foundry Engineering, vol. 10, p. 67-72.

[4] Linder, J., Axelsson, M., Nilsson, H. (2006). The influence of porosity on the fatigue life for sand and permanent mould cast aluminium. International Journal of Fatigue, vol. 28, p. 1752-1758, DOI:10.1016/j. ijfatigue.2006.01.001.

[5] Wang, Q.G. (2001). Fatigue behaviour of A356/357 aluminum cast alloys. Part II - Effect of micro structural constituents. Journal of Light Metals, p. 8597, DOI:10.1016/S1471-5317(00)00009-2.

[6] Manson, S.S. (1953). Behaviour of Material under Conditions of Thermal Stress. Technical note no. 2933. National Advisory Committee for Aeronautics, Cleveland.

[7] Coffin, L.F. (1954). A study of cyclic- thermal stresses in a ductile metal. ASME, vol. 76, p. 931-950.

[8] Rice, J.A. (2007). Mathematical Statistics and Data Analysis, $3^{\text {rd }}$ ed. Thompson Brooks/Cole, Belmont.

[9] Sharma, S. (1996). Applied Multivariate Techniques. John Wiley \&Sons, Hoboken.

[10] Tabachnick, B., Fidell, L.S. (2007). Using Multivariate Statistics, $5^{\text {th }}$ ed. Pearson, Essex.

[11] ASTM. E 606-92 (1996). Standard practice for straincontrolled fatigue testing. ASTM International, West Conshohocken, DOI:10.1520/E0606_E0606M-12.

[12] Janežič, M. (2010). The Influence of Randomness of Damage Model Parameters on Development Prediction of Product Durability. PhD Thesis, University of Ljubljana, Faculty of Mechanical Engineering, Ljubljana. (in Slovene)

[13] Klemenc, J., Fajdiga, M. (2012). Estimating S-N curves and their scatter using a differential ant-stigmergy algorithm. International Journal of Fatigue, vol. 43, p. 90-97, DOI:10.1016/j.ijfatigue.2012.02.015. 